\title{
Enhanced surveillance of invasive meningococcal disease in Canada, 2006-2011
}

\author{
Li Y $A^{1^{\star}}$, Tsang $R^{2}$, Desai $S^{1}$ and Deehan $H^{1}$ \\ 1 Public Health Agency of Canada, Centre for Immunization and Respiratory Infectious Diseases, Ottawa, Ontario \\ 2 Public Health Agency of Canada, National Microbiology Laboratory, Winnipeg, Manitoba \\ * Corresponding author: y.anita.li@phac-aspc.gc.ca
}

\begin{abstract}
Objective: The purpose of this report is to describe the epidemiology of invasive meningococcal disease (IMD) in Canada from 2006 to 2011.

Methods: Data from the Enhanced Invasive Meningococcal Disease Surveillance System and national population estimates were selected for descriptive and inferential analyses. The geographic, demographic, seasonal and subtype distributions as well as clinical characteristics of the IMD cases were examined. Incidence and mortality rates were calculated per 100,000 population per year; 95\% confidence intervals (Cl) were calculated for rate comparison. The direct method was used for age standardization. Proportions were compared using the chisquared test at a $p<0.05$ significance level.

Results: During the study period, the mean incidence rates of IMD were 0.58 (total), 0.33 (serogroup B), 0.07 (serogroup C), 0.03 (serogroup $\mathrm{W}-135$ ) and 0.10 (serogroup $\mathrm{Y}$ ). The median age for serogroups $\mathrm{B}, \mathrm{C}, \mathrm{W}-135$ and $Y$ was 16, 43, 38 and 47 years respectively. The mean age-specific incidence rates among infants under 1 year of age (7.35, Cl: 5.38-9.32) and children from 1 to 4 years of age $(1.89, \mathrm{Cl}: 1.54-2.24)$ were significantly higher than those in any other age group. The mean case fatality ratio was $8.1 \%$ (range $4.3 \%-14.3 \%$ ). The average number of cases that occurred per month was significantly higher $(p<0.0001)$ in winter (18 cases) than in summer $(12$ cases).

Conclusion: IMD is still endemic in Canada. Although individuals at any age can be affected, infants under 1 year of age are at the greatest risk, followed by children aged 1-4 years and individuals aged 15-19 years. Following the implementation of routine childhood immunization programs with monovalent meningococcal $\mathrm{C}$ conjugate vaccines (MenC) in all provinces and territories (beginning in 2007), the incidence of serogroup $C$ has decreased significantly over the study period and is now at an all-time low. Serogroup B is the leading cause of IMD, and diseases of serogroup $\mathrm{W}-135$ and $Y$ have stabilized at relatively lower incidence rates. With the addition of immunization programs using quadrivalent conjugate meningococcal vaccines (MCV4), we would expect further reductions in the incidence of meningococcal infection in Canada.
\end{abstract}

\section{Introduction}

Invasive meningococcal disease (IMD) is caused by Neisseria meningitidis, a gram-negative bacterium. Neisseria are classified into subtypes according to the immunological reactivity of their polysaccharide capsule (1). The illness of IMD is often very rapidly progressive and typically presents as sepsis and/or meningitis (2). IMD has been nationally notifiable in Canada since 1924 (3) through the Canadian Notifiable Diseases Surveillance System, which provides only basic epidemiological data. In Canada, IMD cases caused by serogroups A and C were most frequently identified in cases before 1975 and serogroup B predominated between 1975 and 1985 (4). Since 1985, both the total number of IMD cases and the number of serogroup $C$ cases increased and peaked in $1992(5,6)$. Therefore, the Enhanced Invasive Meningococcal Disease Surveillance System (eIMDSS) was established in 1992 in Canada, capturing epidemiological and laboratory-linked data. Routine immunization programs for meningococcal $\mathrm{C}$ conjugate vaccine (MenC) in infants, recommended by the National Advisory Committee on Immunization (NACl) (6), have been introduced in Canada since 2002 (7). From 2002 to 2005, when only some Canadian jurisdictions had implemented MenC programs, the incidence of serogroup $\mathrm{C}$ disease 
decreased by $43 \%$ compared with the period of 1995-2001 (8). Information on IMD in Canada has been published by the Public Health Agency of Canada (the Agency) since 1979 (4,8-18). The last surveillance report published by the Agency described IMD in Canada from 2004 to 2005 (8). The following report describes the epidemiology of IMD in Canada from January 1, 2006, to December 31, 2011.

\section{Methods}

\section{Surveillance data}

This report is based on IMD data extracted from eIMDSS, with disease onset between January 1, 2006, and December 31, 2011. According to the national case definition (3) the surveillance during this period captured both confirmed and probable cases. Provincial and territorial departments of health report non-nominal epidemiological data to the Agency on all cases of IMD meeting the national case definition. Provincial and territorial public health and/or hospital laboratories send $N$. meningitidis isolates to the National Microbiology Laboratory for confirmation of serogroup and further bacteriologic analyses. These analyses consist of serotyping and subtyping for all isolates, multilocus enzyme electrophoresis or multilocus sequence typing (MLST) for all serogroup C isolates (16) and, more recently, MLST on isolates for outbreak or special analysis. Probabilistic matching was conducted to link epidemiological and laboratory data using the following variables: province/territory, date of birth or age, sex, onset date and serogroup.

Population estimates for the provinces and territories were obtained from Statistics Canada and were based on 2006 Census data (19). The 1991 Canadian population was chosen as the standard population for age standardization. The population distribution is based on the final postcensal estimates for July 1, 1991, Canadian population, adjusted for census undercoverage. The age distribution of the population has been weighted and normalized (20).

The data used in this study come from public health surveillance, which is exempt from research ethics board approval.

\section{Statistical analysis}

The geographic, demographic, seasonal and subtype distributions as well as clinical characteristics of the IMD cases were examined. Incidence rates were calculated using 2006-2011 data from eIMDSS and population estimates. All incidence rates and mortality rates are per 100,000 population per year. The direct method was used for calculating age-standardized rates. Cases with missing age were excluded from the age standardization and all age-related analyses. Confidence intervals $(\mathrm{Cls})$ for incidence rates were calculated at the $95 \%$ confidence level. Cls of age-standardized rates were calculated according to the method based on the gamma distribution (21). Cls of crude and age-specific incidence rates were calculated using methods based on the Poisson distribution for case counts less than 100 and the normal distribution for case counts equal to and greater than 100. Proportions were compared using the chi-squared test, and the significance level was selected at $\mathrm{p}<0.05$. For seasonal analysis, winter was defined as November to April and summer as May to October. Descriptive and inferential analyses were conducted using Microsoft Excel 2010 and SAS EG 5.1.

\section{Results}

\section{Overview}

From 2006 to 2011, a total of 1,174 IMD cases were reported in Canada, ranging from 154 to 229 cases per year (Figure 1). With the exception of 21 cases for which information was missing, 1,121 cases $(97 \%)$ were laboratory confirmed and $32(3 \%)$ were probable cases. Of laboratory-confirmed cases, $777(68 \%)$ were positive by bacterial culture, $111(10 \%)$ by polymerase chain reaction (PCR) and $44(4 \%)$ by both culture and PCR; for the remainder the laboratory method was not specified. 
Figure 1: Number of IMD cases and overall incidence rates (per 100,000 population) in Canada by year, 2006-2011*

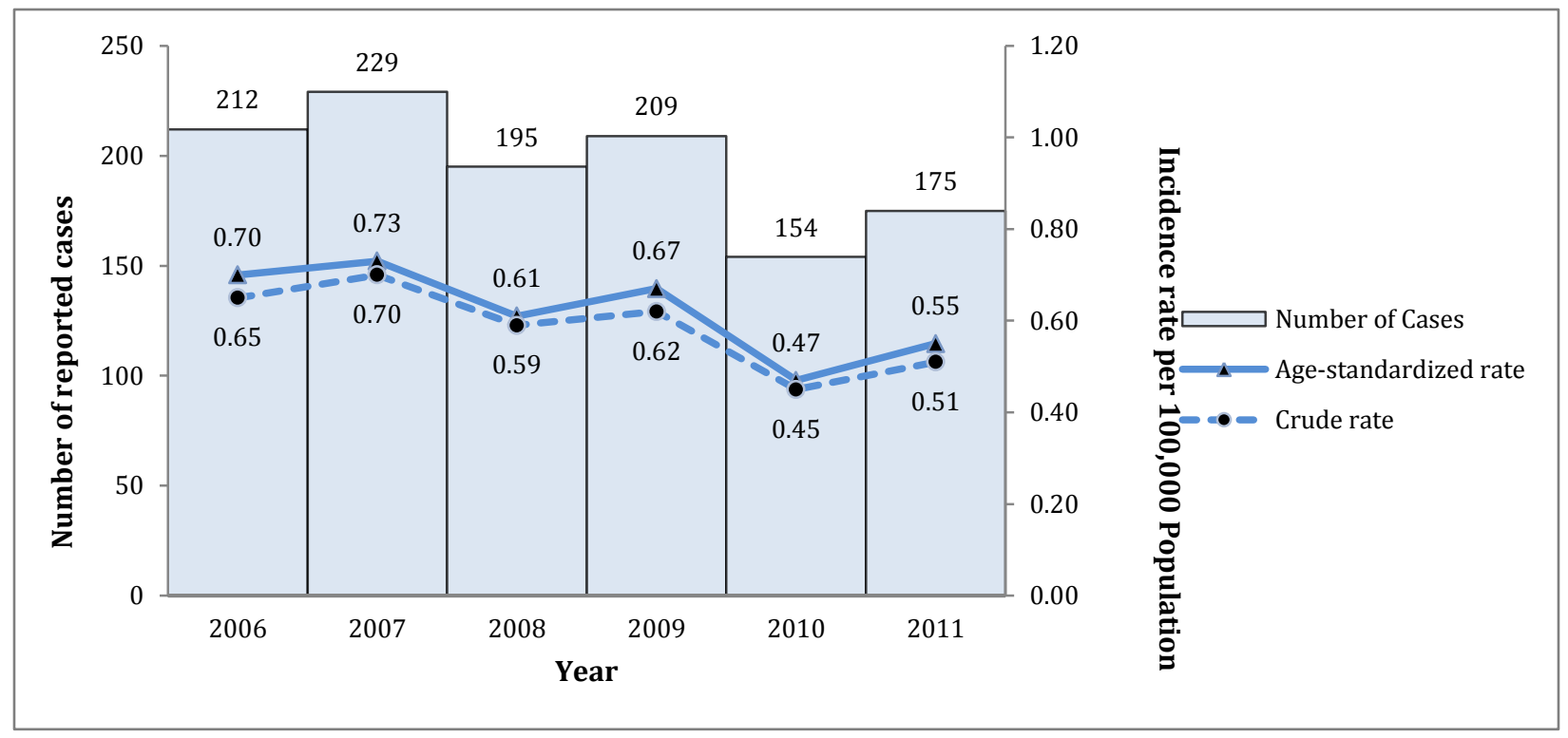

*Three cases without age information were excluded in the calculation of age-standardized rates.

Three cases without age information were excluded for age-standardization and other age-related analyses.

Table 1 provides detailed crude and age-standardized incidence rates. The mean crude and age-standardized incidence rates were 0.58 and 0.60 respectively. The age-standardized incidence rate of IMD in $2010(0.47, \mathrm{Cl}$ : 0.39-0.54) was significantly lower than in 2006 (0.70, Cl: 0.61-0.80), 2007 (0.73, Cl: 0.63-0.82) and 2009 (0.67, $\mathrm{Cl}$ : 0.58-0.76). There were no significant differences identified in other year comparisons. Because of the small number of IMD cases of serogroup A, X, Z and 29E, they were combined as serogroup "other" for further analyses.

Table 1: Crude and age-standardized incidence rates (per 100,000 population) of IMD in Canada by serogroup and year, 2006-2011*

\begin{tabular}{|c|c|c|c|c|c|c|c|c|}
\hline \multirow{2}{*}{ Type of rate } & \multirow{2}{*}{ Serogroup } & \multicolumn{6}{|c|}{ Year } & \multirow{2}{*}{$\begin{array}{c}\text { Mean }(95 \% \mathrm{Cl}) \\
2006-2011\end{array}$} \\
\hline & & 2006 & 2007 & 2008 & 2009 & 2010 & 2011 & \\
\hline \multirow[t]{8}{*}{ Crude } & Total & 0.65 & 0.70 & 0.59 & 0.62 & 0.45 & 0.51 & $0.58(0.49-0.68)$ \\
\hline & B & 0.35 & 0.40 & 0.29 & 0.37 & 0.27 & 0.31 & $0.33(0.28-0.38)$ \\
\hline & C & 0.13 & 0.09 & 0.09 & 0.06 & 0.03 & 0.01 & $0.07(0.02-0.12)$ \\
\hline & W-135 & 0.02 & 0.04 & 0.04 & 0.04 & 0.02 & 0.03 & $0.03(0.02-0.04)$ \\
\hline & $\mathbf{Y}$ & 0.08 & 0.11 & 0.11 & 0.09 & 0.08 & 0.10 & $0.10(0.09-0.11)$ \\
\hline & Other $^{\dagger}$ & 0.006 & 0.006 & 0.003 & 0.018 & 0.006 & 0.012 & $0.008(0.003-0.014)$ \\
\hline & Non-groupable & 0.006 & 0.006 & 0.006 & 0.003 & 0.006 & 0.003 & $0.005(0.003-0.007)$ \\
\hline & Unknown & 0.06 & 0.05 & 0.04 & 0.04 & 0.03 & 0.03 & $0.04(0.03-0.05)$ \\
\hline \multirow[t]{4}{*}{$\begin{array}{l}\text { Age- } \\
\text { standardized }\end{array}$} & Total & 0.70 & 0.73 & 0.61 & 0.67 & 0.47 & 0.55 & $0.60(0.47-0.73)$ \\
\hline & B & 0.40 & 0.44 & 0.33 & 0.40 & 0.24 & 0.36 & $0.36(0.29-0.43)$ \\
\hline & $\mathbf{C}$ & 0.14 & 0.09 & 0.08 & 0.06 & 0.03 & 0.01 & $0.07(0.02-0.11)$ \\
\hline & W-135 & 0.02 & 0.04 & 0.04 & 0.04 & 0.02 & 0.03 & $0.03(0.02-0.04)$ \\
\hline
\end{tabular}




\begin{tabular}{l|c|l|l|l|l|l|l|r}
\hline \multirow{2}{*}{ Type of rate } & \multirow{2}{*}{ Serogroup } & \multicolumn{4}{|c}{ Year } & \multicolumn{2}{c}{ Mean (95\% CI) } \\
\cline { 3 - 9 } & & $\mathbf{2 0 0 6}$ & $\mathbf{2 0 0 7}$ & $\mathbf{2 0 0 8}$ & $\mathbf{2 0 0 9}$ & $\mathbf{2 0 1 0}$ & $\mathbf{2 0 1 1}$ & $\mathbf{2 0 0 6 - 2 0 1 1}$ \\
\hline & $\mathbf{Y}$ & 0.08 & 0.10 & 0.10 & 0.09 & 0.07 & 0.09 & $0.09(0.08-0.10)$ \\
\hline & Other $^{\dagger}$ & 0.005 & 0.005 & 0.004 & 0.013 & 0.005 & 0.011 & $0.007(0.003-0.011)$ \\
\hline & Non-groupable & 0.007 & 0.006 & 0.007 & 0.002 & 0.005 & 0.002 & $0.005(0.003-0.007)$ \\
\hline & Unknown & 0.06 & 0.05 & 0.04 & 0.03 & 0.02 & 0.02 & $0.04(0.02-0.05)$ \\
\hline
\end{tabular}

* A total of 82 cases with serogroup missing were excluded for the calculation of crude incidence rates. A total of 83 cases with either age or serogroup missing were excluded for the calculation of age-standardized incidence rates. tOther: Serogroup A, X, Z and 29E.

\section{Geographic distribution}

As shown in Figure 2, IMD cases were reported in all provinces and territories in Canada but not in every year during the study period. Nunavut had the highest age-standardized incidence rate, of 4.09, in 2006. Because Prince Edward Island and the three territories (Yukon, Nunavut and the Northwest Territories) had reported cases in only one or two years and the populations in these jurisdictions are small, it is not appropriate to compare their mean age-standardized incidence rate with the rates of other provinces. The mean age-standardized incidence rate for Quebec (1.12, Cl: 0.95-1.29) was significantly higher than for any other province except Newfoundland and Labrador (1.08, Cl: 0.53-1.64) and New Brunswick (0.87, Cl: 0.57-1.17). Among other province comparisons, the mean age-standardized incidence rates varied but not significantly. Figure 2: Age-standardized incidence rates (per 100,000 population) of IMD in Canada by
province/territory and year, 2006-2011*

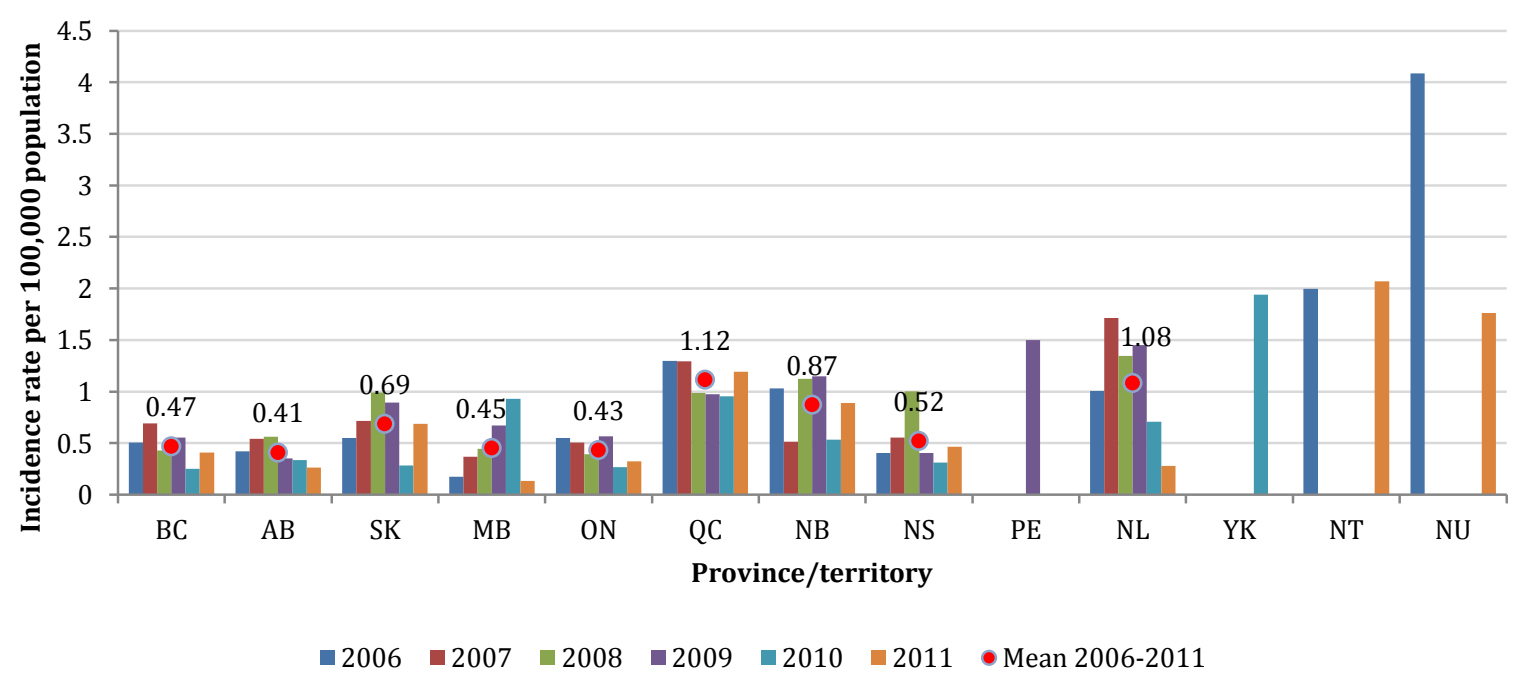

*A total of 3 cases without age information were excluded. Because Prince Edward Island and the three territories (Yukon, Nunavut and the Northwest Territories) had reported cases only in one or two years and the populations in these jurisdictions are small, it is not appropriate to compare their mean age-standardized incidence rate with the rates of other provinces. Therefore, mean age-standardized incidence rates for these four jurisdictions were not displayed.

\section{Demographic distribution}

The age of all reported cases ranged from less than 1 month to 98 years, with a median age of 20 years. The range of median ages was 18-23 years across the study period. The age distribution varied among different serogroups of IMD cases (Figure 3). The median age for serogroups B, C, W-135 and $Y$ was 16, 43, 38 and 47 years respectively. While constituting only $6 \%$ of the total population, children under 5 years accounted for $37 \%$ of serogroup B cases, $31 \%$ of serogroup W-135 cases but only $9 \%$ of serogroup $C$ and $10 \%$ of $Y$ cases. People over 
40 years accounted for the majority of serogroup C (51\%) and $\mathrm{Y}(59 \%)$ cases, nearly half of serogroup W-135 cases (48\%) but only $21 \%$ of serogroup B cases.

Figure 3: Age distribution of IMD cases by serogroup, 2006-2011*

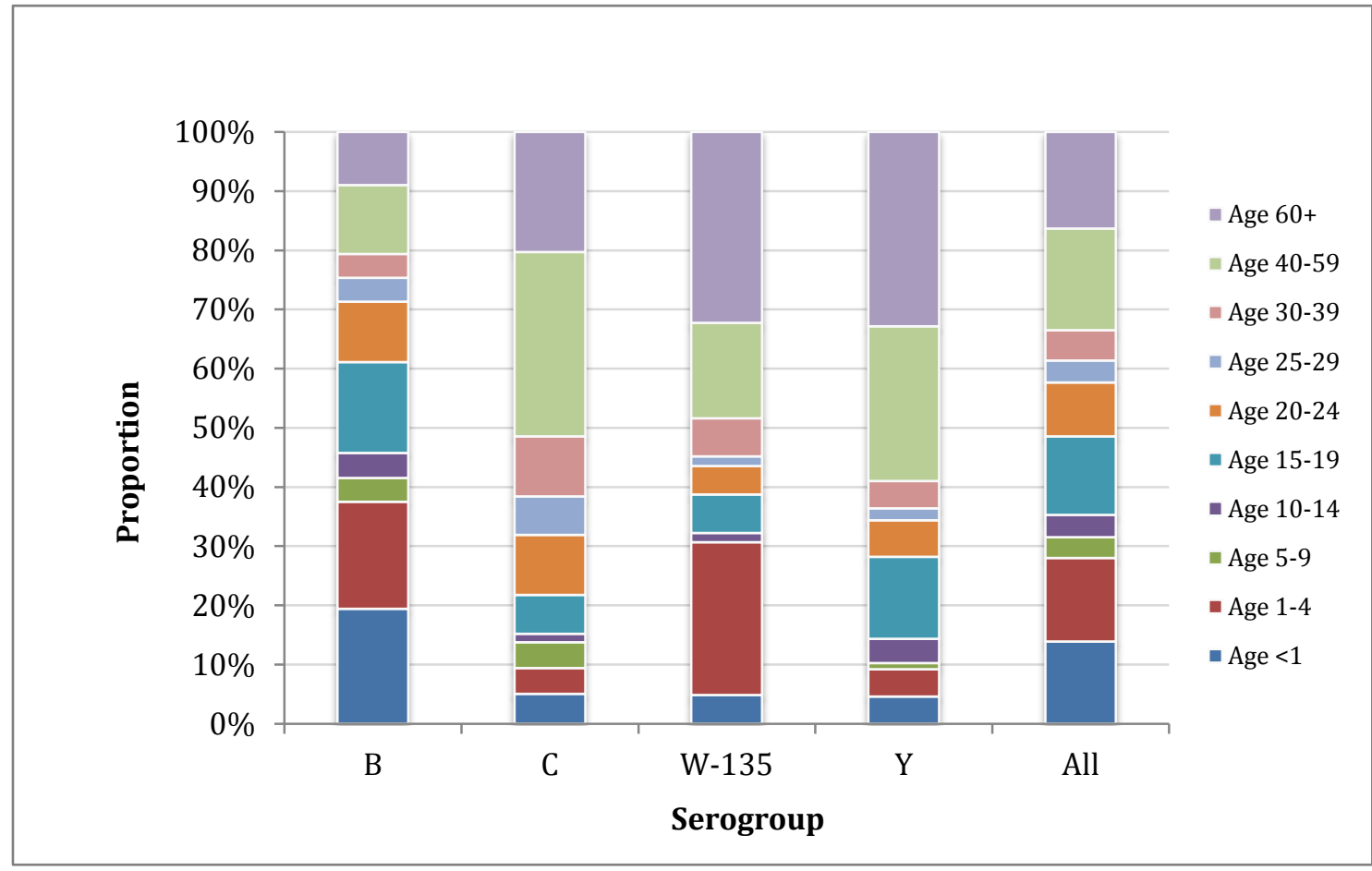

*A total of 83 cases with either age or serogroup missing were excluded.

As shown in Table 2, the mean age-specific incidence rates among infants under 1 year of age (7.35, Cl: 5.389.32) and children from 1 to 4 years of age (1.89, $\mathrm{Cl}$ : 1.54-2.24) were significantly higher than those of any other age group. Adolescents from 15 to 19 years of age had the third highest incidence rate (1.17, $\mathrm{Cl}: 1.00-1.34)$ except age 20 to 24 years $(0.77, \mathrm{Cl}: 0.48-1.05)$.

Table 2: Age-specific incidence rates (per 100,000 population) of IMD in Canada by year, 2006-2011*

\begin{tabular}{|c|c|c|c|c|c|c|c|}
\hline \multirow{2}{*}{ Age group } & \multicolumn{6}{|c|}{ Year } & \multirow{2}{*}{$\begin{array}{l}\text { Mean }(95 \% \mathrm{Cl}) \\
2006-2011\end{array}$} \\
\hline & 2006 & 2007 & 2008 & 2009 & 2010 & 2011 & \\
\hline All & 0.65 & 0.70 & 0.59 & 0.62 & 0.45 & 0.51 & $0.58(0.49-0.68)$ \\
\hline$<1$ & 7.71 & 6.94 & 6.97 & 10.57 & 4.75 & 7.15 & 7.35 (5.38-9.32) \\
\hline $1-4$ & 1.81 & 1.92 & 1.61 & 2.32 & 1.46 & 2.22 & $1.89(1.54-2.24)$ \\
\hline 5-9 & 0.44 & 0.78 & 0.22 & 0.50 & 0.11 & 0.22 & $0.38(0.12-0.63)$ \\
\hline $10-14$ & 0.43 & 0.34 & 0.45 & 0.35 & 0.26 & 0.37 & $0.37(0.30-0.44)$ \\
\hline $15-19$ & 1.40 & 1.30 & 1.20 & 1.02 & 0.99 & 1.09 & $1.17(1.00-1.34)$ \\
\hline $20-24$ & 1.24 & 0.92 & 0.70 & 0.60 & 0.63 & 0.50 & $0.77(0.48-1.05)$ \\
\hline $25-29$ & 0.27 & 0.54 & 0.35 & 0.30 & 0.21 & 0.25 & $0.32(0.20-0.44)$ \\
\hline $30-39$ & 0.40 & 0.24 & 0.20 & 0.18 & 0.17 & 0.13 & $0.22(0.12-0.32)$ \\
\hline $40-59$ & 0.27 & 0.45 & 0.39 & 0.34 & 0.36 & 0.20 & $0.33(0.24-0.43)$ \\
\hline $60+$ & 0.55 & 0.60 & 0.55 & 0.51 & 0.32 & 0.51 & $0.51(0.40-0.61)$ \\
\hline
\end{tabular}

*Three cases without age information were excluded. 
During the study period, the mean age-standardized incidence rates were 0.63 ( $\mathrm{Cl}: 0.50-0.75)$ among males and 0.57 (Cl: 0.42-0.73) among females, a difference that was not significant.

\section{Seasonality}

A total of 99 cases without onset date were excluded for seasonality analysis. The occurrence of IMD cases peaked in winter months. The average number of cases that occurred per month was significantly higher $(p<0.0001)$ in winter (18 cases) than in summer (12 cases).

\section{Subtype distribution}

\section{Serogroup}

A total of 1,092 cases (93\%) had serogroup information. The number of reported cases was highest for serogroup B (669), followed by serogroups $Y(196), C$ (138) and W-135 (62). Cases of these four serogroups accounted for $91 \%$ of total reported IMD cases. IMD cases caused by other serogroups were rare and included serogroups $29 \mathrm{E}$ ( 7 cases), A ( 3 cases), X ( 2 cases) and Z ( 2 cases); 10 cases were non-groupable and 3 non-specified.

Table 1 and Figure 4 provide the detailed serogroup distribution of IMD cases in Canada. The mean agestandardized incidence rate of serogroup $B$ was the highest (0.36, Cl: 0.29-0.43) of all serogroups. The adjusted incidence rate of serogroup B in $2010(0.24, \mathrm{Cl}: 0.19-0.30)$ was significantly lower than that in 2006, 2007 and 2009. The proportion of serogroup B cases among all IMD cases was the highest every year during the study period and ranged from $50 \%$ to $62 \%$ of cases. The age-standardized incidence rate of serogroup $\mathrm{C}$ decreased significantly from 0.14 (Cl: $0.10-0.19)$ in 2006 to 0.01 (0.004-0.04) in 2011. The proportion of serogroup $\mathrm{C}$ cases was significantly higher than serogroup W-135 from $2006(p<0.001)$ to $2008(p=0.02)$; the difference was no longer significant from 2009 to 2011 . The proportion of serogroup $\mathrm{C}$ cases became significantly lower than serogroup $Y$ in $2010(p=0.004)$ and $2011(p<0.0001)$. Although the proportion of serogroup $Y$ cases increased gradually from $13 \%$ in 2006 to $21 \%$ in 2011 , the age-standardized incidence rates for this group did not change significantly over the study period (range 0.07 to 0.10 ). The proportion of serogroup $Y$ remained significantly higher than that of serogroup W-135 from 2006 to 2011. The age-standardized incidence rate of serogroup W-135 ranged 0.02 to 0.04 , and no significant difference was identified over the 6 years.

Figure 4: Proportion of serogroups among all reported cases in Canada by year, 2006-2011*

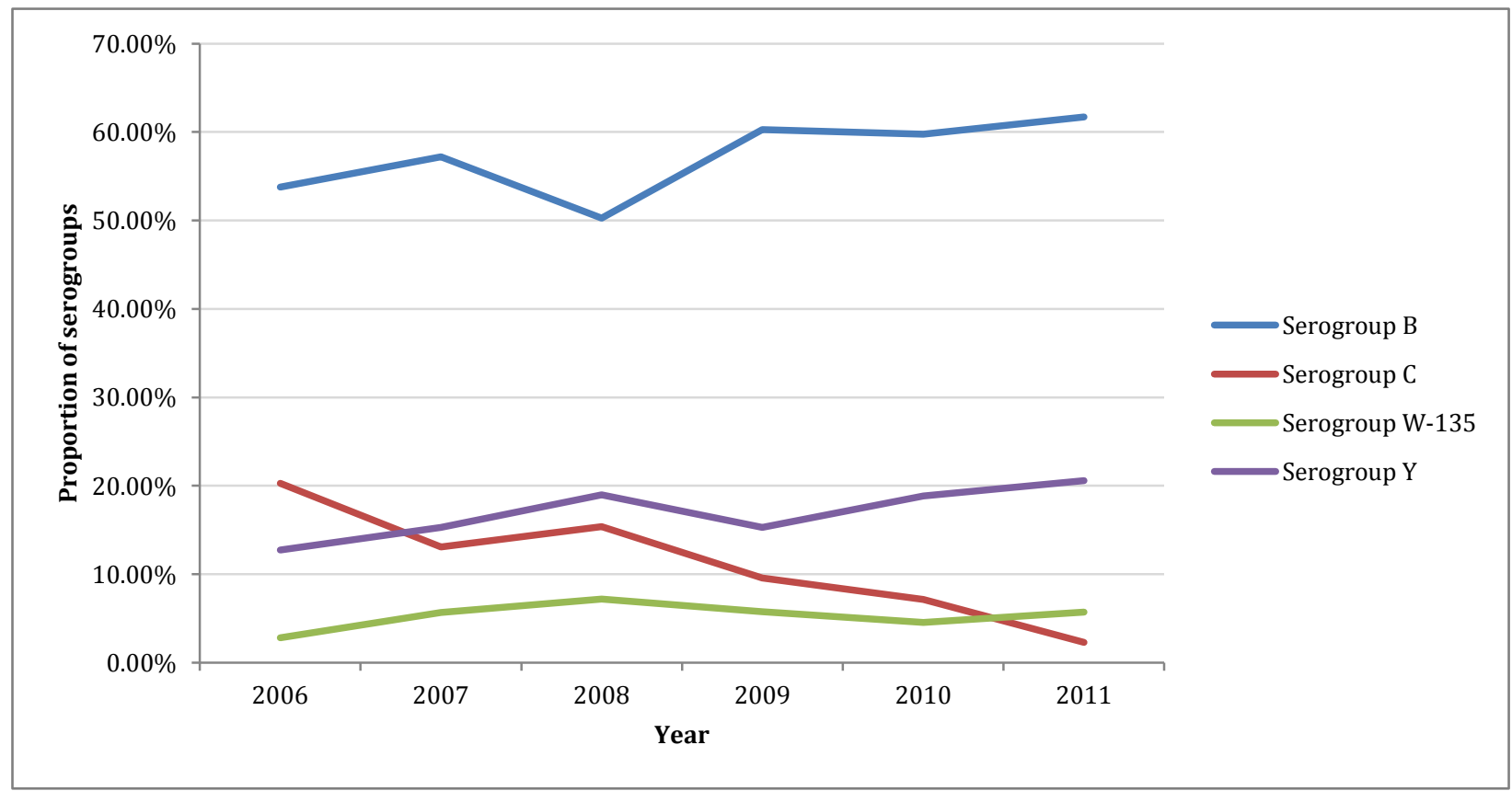

*A total of 82 cases without serogroup information were excluded. 


\section{Serotypes and serosubtypes}

Serotype and serosubtype analysis included $73 \%$ of the IMD cases $(862 / 1,174)$, for which information was available. Of 507 serogroup B cases, $32 \%$ were serotype 17 , followed by $22 \%$ for serotype $4 ; 14 \%$ were nontypable. The most common antigenic formulas for serogroup B were B:17:P1.19 (25\%) and B:4:P1.4 (9\%). Of 113 serogroup $\mathrm{C}$ cases, the majority were serotype $2 \mathrm{a}(83 \%)$, and $12 \%$ were non-typable. The most common antigenic formula for serogroup C was C:2a:P1.2,5 (30\%). Of 163 serogroup Y cases, 29\% were non-typable; $28 \%$ were serotype $2 \mathrm{c}$ and $25 \%$ were serotype 14,19 . The most common antigenic formulas for serogroup $Y$ cases were Y:14,19:P1.- (17\%) and Y:2c:P1.2,5. (16\%). Of 60 serogroup W-135 cases, 57 (95\%) were nontypable, and the remaining 3 cases were serotype $19,2 \mathrm{a}$ and $2 \mathrm{c}$.

\section{Clinical characteristics}

\section{Clinical diagnoses}

During the study period, of 375 cases with clinical diagnoses, $46 \%$ had meningitis and $45 \%$ had septicemia, with or without other manifestations. Six cases had septic arthritis.

\section{Fatality}

A total of 94 deaths occurred during the study period. The case fatality ratios (CFRs) ranged from $4.3 \%$ in 2010 to $14.3 \%$ in 2011 , with an average of $8.1 \%$. The median age of fatal cases by year ranged from 17 to 26 years, except in 2008, when it was 45 years. There was no statistical age difference identified ( $\mathrm{p}=0.11)$. CFRs of serogroups B, C, W-135 and $\mathrm{Y}$ were 5.5\% (Cl: 4.0-7.5\%), 14.5\% (Cl: 9.6-21.3\%), 8.1\% (Cl: 3.5-17.5\%) and $11.2 \%(\mathrm{Cl}: 7.5-16.4 \%)$ respectively. There was a significant difference in CFRs between serogroup $B$ and serogroup $\mathrm{C}$.

\section{Mortality}

The age-standardized mortality rate of IMD ranged from 0.02 in 2010 to 0.07 in 2011, with a mean of 0.05 . Infants had the highest morality rate, of $0.62(\mathrm{Cl}: 0.30-0.96)$.

\section{Discussion}

This study demonstrates that IMD is still endemic in Canada. Although individuals at any age can be affected, infants under 1 year of age are at the greatest risk, followed by children aged 1-4 years and then individuals aged 15-19 years. IMD incidence showed typical seasonal variation, peaking in the winter. In Canada, serogroups B, C, $\mathrm{W}-135$ and $\mathrm{Y}$ were the most commonly reported serogroups. These finding are consistent with previous Canadian data $(8,17,22)$. With the introduction of routine childhood immunization programs and routine adolescent immunization programs (7), not unexpectedly the incidence of serogroup $C$ has decreased significantly over the study period. Since 2007, all Canadian provinces and territories have offered routine childhood immunization programs against meningococcal $\mathrm{C}$ infection as a part of their routine programs (7). Compared with the early implementation period (2002-2005) of MenC immunization programs, the mean incidence of serogroup C decreased by $39 \%$ from 0.18 (8) to 0.07 . Serogroup B is the leading cause of IMD, and diseases of serogroup W135 and $\mathrm{Y}$ have stabilized at relatively lower incidence rates. NACl recommended the use of quadrivalent conjugate meningococcal vaccines (MCV4) for high-risk groups aged 2-55 years in 2007 and routine adolescent immunization programs with either MenC or MCV4 in 2009 (7). During the study period, only a few provinces and territories had implemented MCV4 immunization programs. Therefore, this study is not able to assess the impact of immunization programs for MCV4. Further years of enhanced surveillance data are needed.

Studies of population biology of invasive $N$. meningitidis strains by MLST have revealed a predominant clone of serogroup B ST-269 in the province of Quebec leading to an increase in the incidence of IMD (23-25). In the neighbouring province of Ontario, no similar increase of this strain or cases of disease have been observed $(26,27)$. An unusual shift in the genotype of invasive meningococcal $\mathrm{C}$ strains has also been observed, from predominantly ET15 type to ET-37 (non-ET-15) type (28). The overall case-fatality rate of IMD in Canada remained steady, as in previous years $(8,17)$, and is comparable to that of other nations $(29,30)$. IMD caused by serogroup $C$ was much more severe than disease caused by serogroup $B$ in terms of fatality. The serotype and 
serosubtype distributions of $N$. meningitidis strains in this study were similar to those of cases reported in previous years (8), based on a limited number of samples.

In conclusion, on the basis of national enhanced data, following the implementation of routine childhood immunization programs for MenC in all provinces and territories (beginning in 2007) the incidence of meningococcal $\mathrm{C}$ is now at an all-time low. With the addition of MCV4, we would expect further reductions in the incidence of meningococcal infection in Canada. Future studies with a focus on the impact of various meningococcal vaccination programs in Canada are needed.

\section{Limitations}

The eIMDSS does not receive data from provinces and territories in real time, nor are cases that are reported at the national level linked with laboratory and epidemiological (lab-epi) data. In addition, as there are no formalized data-sharing agreements, some data elements were incomplete. Therefore, it is not possible to determine whether this study has underestimated or overestimated case characteristics. Cases that were part of an outbreak were not identified as such in this study because of the lack of detailed data provided by provinces and territories. The immunization status of cases was not analyzed because over half of the cases had missing immunization history. Because of the unreliability of results based on small numbers, caution should be used when interpreting results such as serogroup-specific fatality ratios, mortality rates and incidence rates based on less than 20 cases.

\section{Notes and acknowledgements}

Data extracted from eIMDSS represent a snapshot at the time of extraction and may differ from those of previous or subsequent publications. We gratefully acknowledge provincial and territorial health ministries and laboratories for providing data to the eIMDSS. We would also like to thank all colleagues from the provincial and territorial health ministries for providing their input to this report.

\section{Conflict of interest}

No conflicts of interests to declare.

\section{References}

(1) Heymann DL. Control of Communicable Diseases Manual. 19th ed. American Public Health Association; 2008. p. 403.

(2) Mandell G, Bennett J, Dolin P. Chapter 211 - Neisseria meningitidis. Mandell, Douglas, and Bennett's Principles and Practice of Infectious Diseases. 7th ed. Philadelphia: Churchill Livingstone; 2009.

(3) Public Health Agency of Canada. Case definitions for communicable diseases under national surveillance. CCDR 2009 November;35(S2).

(4) Varughese PV, Acres S. Meningococcal disease in Canada and serogroup distribution. Can Dis Wkly Rep 1983;9(45):177-80.

(5) Whalen CM, Hockin JC, Ryan A, Ashton F. The changing epidemiology of invasive meningococcal disease in Canada, 1985 through 1992: emergence of a virulent clone of Neisseria meningitidis. JAMA 1995 February 1;273(5):390-394.

(6) National Advisory Committee on Immunization (NACI). An Advisory Committee Statement (ACS). Statement on recommended use of meningococcal vaccines. Can Commun Dis Rep 2001 Oct 15;27:2-36.

(7) National Advisory Committee on Immunization ( $\mathrm{NACl}$ ). An update on the invasive meningococcal disease and meningococcal vaccine conjugate recommendations. An Advisory Committee Statement (ACS). Can Commun Dis Rep 2009 Apr;35(ACS-3):1-40. 
(8) Enhanced surveillance of invasive meningococcal disease in Canada: 1 January, 2004, through 31 December, 2005. Can Commun Dis Rep 2007 Jun 1;33(10):1-15.

(9) Ashton FE, Ryan JA. Association of serotypes 2A and 2B with meningococcal disease in Canada. Can Dis Wkly Rep 1989 Mar 18;15(11):63-65.

(10) Varughese PV, Carter AO. Meningococcal disease in Canada. Surveillance summary to 1987. Can Dis Wkly Rep 1989 Apr 29;15(17):89-96.

(11) Meningococcal disease - Canada, 1991. Can Dis Wkly Rep 1991 Dec 28;17(52):293.

(12) Ashton FE, Mancino L, Ryan AJ, Poolman JT, Abdillahi H, Zollinger WD. Serotypes and subtypes of Neisseria meningitidis serogroup B strains associated with meningococcal disease in Canada, 1977-1989. Can J Microbiol 1991 Aug;37(8):613-617.

(13) Bureau of Communicable Disease Epidemiology, Laboratory Centre for Disease Control. Guidelines for control of meningococcal disease. Can Commun Dis Rep 1994 Feb 15;20(3):17-27.

(14) Squires SG, Pelletier L, Mungai M, Tsang R, Collins F, Stoltz J. Invasive meningococcal disease in Canada, 1 January 1997 to 31 December 1998. Can Commun Dis Rep 2000 Nov 1;26(21):177-182.

(15) Squires SG, Deeks SL, Tsang RS. Enhanced surveillance of invasive meningococcal disease in Canada: 1 January, 1999, through 31 December, 2001. Can Commun Dis Rep 2004 Feb 1;30(3):17-28.

(16) Law DK, Stoltz J, Henderson AM, Tsang RS. Antigenic and genetic characterization of serogroup C meningococci isolated from invasive meningococcal disease cases in Canada from 1999 to 2003. Can J Microbiol 2005 Jul;51(7):523-530.

(17) Enhanced surveillance of invasive meningococcal disease in Canada: 1 January, 2002, through 31 December, 2003. Can Commun Dis Rep 2006 Apr 15;32(8):97-107.

(18) Tsang RS, Law DK, Henderson AM, Blake ML, Stoltz J. Increase in serogroup C meningococcal disease in Canada is associated with antigenic changes in the protein antigens of the ET-15 clone of Neisseria meningitidis. J Infect Dis 2006 Dec 15;194(12):1791-2; author reply 1792-3.

(19) Statistics Canada, Demography Division, Demographic Estimates Section. July Population Estimates, 2011. Final Intercensal Estimate.

(20) Statistics Canada. Table105-0503 - Health indicator profile, age-standardized rate, annual estimates, by sex, Canada, provinces and territories, occasional, CANSIM (database). 2013; http://www5.statcan.gc.ca/cansim/pick-choisir?lang=eng\&p2=33\&id=1050503\#F57. Accessed 1/13, 2014.

(21) Fay MP, Feuer EJ. Confidence intervals for directly standardized rates: a method based on the gamma distribution. Stat Med 1997 Apr 15;16(7):791-801.

(22) Bettinger JA, Scheifele DW, Le Saux N, Halperin SA, Vaudry W, Tsang R, et al. The impact of childhood meningococcal serogroup C conjugate vaccine programs in Canada. Pediatr Infect Dis J 2009 Mar;28(3):220224.

(23) Law DK, Lorange M, Ringuette L, Dion R, Giguere M, Henderson AM, et al. Invasive meningococcal disease in Quebec, Canada, due to an emerging clone of ST-269 serogroup B meningococci with serotype antigen 17 and serosubtype antigen P1.19 (B:17:P1.19). J Clin Microbiol 2006 Aug;44(8):2743-2749.

(24) Zhou J, Lefebvre B, Deng S, Gilca R, Deceuninck G, Law DK, et al. Invasive serogroup B Neisseria meningitidis in Quebec, Canada, 2003 to 2010: persistence of the ST-269 clone since it first emerged in 2003. J Clin Microbiol 2012 May;50(5):1545-1551. 
(25) Gilca R, Deceuninck G, Lefebvre B, Tsang R, Amini R, Gilca V, et al. The changing epidemiology of meningococcal disease in Quebec, Canada, 1991-2011: potential implications of emergence of new strains. PLoS One 2012;7(11):e50659.

(26) Dang V, Jamieson FB, Wilson S, Rawte P, Crowcroft NS, Johnson K, et al. Epidemiology of serogroup B invasive meningococcal disease in Ontario, Canada, 2000 to 2010. BMC Infect Dis 2012 Aug 29;12:202-233412-202.

(27) Jamieson FB, Rawte P, Deeks SL, Zhou J, Law DK, Deng S, et al. Genetic and antigenic characterization of invasive endemic serogroup B Neisseria meningitidis from Ontario, Canada, in 2001-2010. J Med Microbiol 2013 Jan;62(Pt 1):46-55.

(28) Zhou J, Jamieson F, Dolman S, Hoang LM, Rawte P, Tsang RS. Genetic and antigenic analysis of invasive serogroup C Neisseria meningitidis in Canada: a decrease in the electrophoretic type (ET)-15 clonal type and an increase in the proportion of isolates belonging to the ET-37 (but not ET-15) clonal type during the period from 2002 to 2009. Can J Infect Dis Med Microbiol 2012 Fall;23(3):e55-9.

(29) Thigpen MC, Whitney CG, Messonnier NE, Zell ER, Lynfield R, Hadler JL, et al. Bacterial meningitis in the United States, 1998-2007. N Engl J Med 2011 May 26;364(21):2016-2025.

(30) Hellenbrand W, Elias J, Wichmann O, Dehnert M, Frosch M, Vogel U. Epidemiology of invasive meningococcal disease in Germany, 2002-2010, and impact of vaccination with meningococcal C conjugate vaccine. J Infect 2013 Jan;66(1):48-56. 